IJMMS 29:1 (2002) 7-15

PII. S0161171202011018

http://ijmms.hindawi.com

(c) Hindawi Publishing Corp.

\title{
THE GEOMETRY OF AUTONOMOUS METRICAL MULTI-TIME LAGRANGE SPACE OF ELECTRODYNAMICS
}

\author{
MIRCEA NEAGU
}

Received 12 October 2000

\begin{abstract}
The aim of this paper is to create a large geometrical background for the study of important branch of physics: electrodynamics, bosonic strings theory, magneto-hydrodynamics, and so forth. The geometrical construction is realized on the 1-jet fibre bundle $J^{1}(T, M)$ and is produced by a given quadratic multi-time Lagrangian function $L$. The Riemann-Lagrange geometry of the space $E D M L_{p}^{n}=\left(J^{1}(T, M), L\right)$, in the sense of $d$-connections, torsion and curvature $d$-tensors, allows the construction of a natural generalized multi-time field theory on $E D M L_{p}^{n}$, in the sense of generalized Maxwell and Einstein equations.
\end{abstract}

2000 Mathematics Subject Classification: 53B40, 53C60, 53C80.

Section 1 contains physical and geometrical aspects that motivates us to study the autonomous metrical multi-time Lagrangian space of electrodynamics, denoted $E D M L_{p}^{n}$. Section 2 constructs the canonical nonlinear connection $\Gamma$ and the generalized Cartan canonical $\Gamma$-linear connection of $E D M L_{p}^{n}$. Section 3 describes the generalized Maxwell equations which govern the electromagnetic field of this space. The generalized Einstein equations of the gravitational $h$-potential of the autonomous metrical multi-time Lagrange space are written in Section 4 . The generalized conservation laws of these equations will also be described.

1. Geometrical and physical aspects. In the last thirty years, many geometrical models in Mechanics or Physics were based on the notion of ordinary Lagrangian. Thus, the geometrical concept of Lagrange space was introduced. The differential geometry of the Lagrange spaces is now considerably developed and used in various fields to study the natural processes where the dependence on position, velocity or momentum are involved [2]. We recall that a Lagrange space $L^{n}=(M, L(x, y))$ is defined as a pair which consists of a real, $n$-dimensional manifold $M$ coordinated by $x=\left(x^{i}\right)_{i=\overline{1, n}}$ and a regular Lagrangian $L: T M \rightarrow \mathbb{R}$ (i.e., the fundamental metrical $d$ tensor $g_{i j}(x, y)=(1 / 2)\left(\partial^{2} L / \partial y^{i} \partial y^{j}\right)$ is of rank $n$ and has a constant signature on $T M \backslash\{0\})$. We point out that the Lagrangian $L$ is not necessarily homogeneous with respect to the direction $y=\left(y^{i}\right)_{i=\overline{1, n}}$.

An important and well-known example of Lagrange space comes from electrodynamics. We recall that the Lagrangian $L: T M \rightarrow \mathbb{R}$ which governs the movement law of a particle of mass $m \neq 0$ and electric charge $e$, placed concurrently into a gravitational field and an electromagnetic one, is given by

$$
L(x, y)=m c \varphi_{i j}(x) y^{i} y^{j}+\frac{2 e}{m} A_{i}(x) y^{i}+U(x),
$$


where the semi-Riemannian metric $\varphi_{i j}(x)$ represents the gravitational potentials of the space $M, A_{i}(x)$ are the components of a covector field on $M$ representing the electromagnetic potentials, $U(x)$ is a function on $M$ which is called potential function, and $c$ is the physical constant of light speed. It is obvious that $L$ is a regular Lagrangian and, consequently, the pair $L^{n}=(M, L(x, y))$ is a Lagrange space, which is called the Lagrange space of electrodynamics.

At the same time, there are many problems in Mechanics that rely on the notion of time-dependent Lagrangian. A geometrization of time-dependent Lagrangians was also constructed in [2], the authors developing a classical rheonomic mechanics. From their point of view, a time-dependent Lagrangian is a function $L: \mathbb{R} \times T M \rightarrow \mathbb{R}$, where the product manifold $\mathbb{R} \times T M$ is regarded as a vector bundle over the base space $M$. In this approach, the bundle of configurations of the classical rheonomic mechanics is

$$
\mathbb{R} \times T M \longrightarrow M, \quad\left(t, x^{i}, y^{i}\right) \longrightarrow\left(x^{i}\right),
$$

whose geometrical invariance group is of the form

$$
\tilde{t}=(t), \quad \tilde{x}^{i}=\tilde{x}^{i}\left(x^{j}\right), \quad \tilde{y}^{i}=\frac{\partial \tilde{x}^{i}}{\partial x^{j}} y^{j} .
$$

Obviously, the gauge group (1.3) ignores the temporal reparametrizations, standing out by the absolute character of the temporal coordinate $t$.

In the classical rheonomic mechanics, a central role is played by the time-dependent Lagrangian of classical rheonomic electrodynamics, whose expression is

$$
L(t, x, y)=m c \varphi_{i j}(x) y^{i} y^{j}+\frac{2 e}{m} A_{i}(t, x) y^{i}+U(t, x) .
$$

The differential geometry induced by the classical time-dependent Lagrangian of electrodynamics (1.4) is found in [2].

In contrast, a geometrization of time-dependent Lagrangians, in a relativistic approach, or, in other words, a relativistic rheonomic mechanics, was created by Neagu [4], considering the bundle of configurations represented by the jet fibre bundle of order one

$$
J^{1}(\mathbb{R}, M) \equiv \mathbb{R} \times T M \longrightarrow \mathbb{R} \times M, \quad\left(t, x^{i}, y^{i}\right) \longrightarrow\left(t, x^{i}\right),
$$

whose invariance gauge group is

$$
\tilde{t}=\tilde{t}(t), \quad \tilde{x}^{i}=\tilde{x}^{i}\left(x^{j}\right), \quad \tilde{y}^{i}=\frac{\partial \tilde{x}^{i}}{\partial x^{j}} \frac{d t}{d \tilde{t}^{\prime}} y^{j},
$$

where $\left(t, x^{i}, y^{i}\right)$ are the coordinates on $J^{1}(\mathbb{R}, M)$. It is obvious that the form of this gauge group (1.6) is more general than that used in the classical rheonomic mechanics and emphasizes the relativistic character of the temporal coordinate $t$.

According to Olver terminology [1], the relativistic rheonomic mechanics relies on the notion of Lagrangian $\mathscr{L}$ on $J^{1}(\mathbb{R}, M)$ as a local function on the 1-jet space, which transforms by the rule $\tilde{\mathscr{L}}=\mathscr{L}|d t / d \tilde{t}|$. Like a distinct notion, the concept of Lagrangian function $L: J^{1}(\mathbb{R}, M) \rightarrow \mathbb{R}$ is also involved in relativistic rheonomic mechanics. 
REMARK 1.1. It is important to note the difference between the notions of the Lagrangian used in both relativistic and classical rheonomic mechanics. From this point of view, the reader is invited to compare them, following the expositions done in $[2,4]$.

We emphasize that, in the relativistic rheonomic mechanics, a basic role is played by the following Lagrangian of relativistic rheonomic electrodynamics,

$$
\mathscr{L}=\left[m c \psi^{11}(t) \varphi_{i j}(x) y^{i} y^{j}+\frac{2 e}{m} A_{(i)}^{(1)}(t, x) y^{i}+U(t, x)\right] \sqrt{\left|\psi_{11}\right|},
$$

where $\psi_{11}$ is a semi-Riemannian metric on $\mathbb{R}, A_{(i)}^{(1)}(t, x)$ is a distinguished tensor on $J^{1}(\mathbb{R}, M)$ and $U(t, x)$ is a smooth function on $\mathbb{R} \times M$. The differential geometry generated by this Lagrangian is exposed in [4].

To become general, consider the jet fibre bundle of order one [7]

$$
J^{1}(T, M) \longrightarrow T \times M, \quad\left(t^{\alpha}, x^{i}, x_{\alpha}^{i}\right) \longrightarrow\left(t^{\alpha}, x^{i}\right)
$$

where $T$ is a real, $p$-dimensional manifold coordinated by $t=\left(t^{\alpha}\right)_{\alpha=\overline{1, p}}$, whose physical meaning is that of "multi-time," $M$ is a real, $n$-dimensional "spatial" manifold coordinated by $x=\left(x^{i}\right)_{i=\overline{1, n}}$, while the coordinates $x_{\alpha}^{i}$ have the meaning of partial directions or partial derivatives.

We should like to underline that the jet fibre bundle of order one $J^{1}(T, M)$ is a basic object in the study of classical and quantum field theories [8]. From a physical point of view, the 1-jet fibre bundle $J^{1}(T, M) \rightarrow T \times M$ can be regarded as a bundle of configurations, in mechanics terms, because, considering the particular case of the temporal manifold $T=\mathbb{R}$ (i.e., the usual time axis represented by the set of real numbers), we recover the bundle of configurations (1.5) from relativistic rheonomic mechanics.

It is well known that a lot of problems in Physics and Variational Calculus rely on multi-time Lagrangian functions $L$ depending on first order partial derivatives, which are viewed as functions defined on the total space of the 1 -jet fibre bundle $J^{1}(T, M)$. A well-known example, which comes from Physics, is given by the "energy" Lagrangian function $L$ used in the Polyakov model of bosonic strings,

$$
L\left(t^{\gamma}, x^{k}, x_{\gamma}^{k}\right)=\frac{1}{2} \psi^{\alpha \beta}(t) \varphi_{i j}(x) x_{\alpha}^{i} x_{\beta}^{j},
$$

where $\psi^{\alpha \beta}(t)$ (resp., $\varphi_{i j}(x)$ ) is a semi-Riemannian metric on the manifold $T$ (resp., $M)$. We recall that the extremals of the Lagrangian $\mathscr{L}=L \sqrt{|\psi|}$ are exactly the harmonic maps between the semi-Riemannian spaces $(T, \psi)$ and $(M, \varphi)$.

In this context, a geometrization of a multi-time Lagrangian function $L: J^{1}(T, M) \rightarrow$ $\mathbb{R}$ is imposed. Recently, a differential geometry, attached to certain multi-time Lagrangian functions, was created in [6]. In order to present the main concept of this geometry, fix a semi-Riemannian metric $\psi=\psi_{\alpha \beta}\left(t^{\gamma}\right)$ on the temporal manifold $T$. The fundamental geometrical concept used in the geometrization of a multi-time Lagrangian function is that of metrical multi-time Lagrange space, represented by a pair $M L_{p}^{n}=\left(J^{1}(T, M), L\right)$ consisting of 1 -jet space and a Kronecker $\psi$-regular multi-time 
Lagrange function $L$, that is, [6]

$$
G_{(i)(j)}^{(\alpha)(\beta)}\left(t^{\gamma}, x^{k}, x_{\gamma}^{k}\right)=\frac{1}{2} \frac{\partial^{2} L}{\partial x_{\alpha}^{i} \partial x_{\beta}^{j}}=\psi^{\alpha \beta}\left(t^{\gamma}\right) \varphi_{i j}\left(t^{\gamma}, x^{k}, x_{\gamma}^{k}\right),
$$

where $\varphi_{i j}\left(t^{\gamma}, x^{k}, x_{\gamma}^{k}\right)$ is a $d$-tensor on $J^{1}(T, M)$, symmetric, of rank $n$ and having a constant signature. We point out that the differential geometry of metrical multi-time Lagrange spaces is now considerably developed in [5, 6].

By a natural extension of previous examples of Lagrangian functions, we can give a very important example of metrical multi-time Lagrange space, considering the general Lagrangian function $L$ which comes from electrodynamics and theory of bosonic strings, namely,

$$
L=m c \psi^{\alpha \beta}(t) \varphi_{i j}(x) x_{\alpha}^{i} x_{\beta}^{j}+\frac{2 e}{m} A_{(i)}^{(\alpha)}(t, x) x_{\alpha}^{i}+U(t, x),
$$

where $A_{(i)}^{(\alpha)}(t, x)$ is a distinguished tensor on $J^{1}(T, M)$ and $U(t, x)$ is a smooth function on $T \times M$.

Now, in order to unify all Lagrangian entities exposed above, we introduce the following geometrical concept.

DEFINITION 1.2. A pair $E D M L_{p}^{n}=\left(J^{1}(T, M), L\right)$ which consists of a jet fibre bundle of order one and a Lagrangian function of the form

$$
L\left(t^{\gamma}, x^{k}, x_{\gamma}^{k}\right)=h^{\alpha \beta}\left(t^{\gamma}\right) g_{i j}\left(x^{k}\right) x_{\alpha}^{i} x_{\beta}^{j}+U_{(i)}^{(\alpha)}\left(t^{\gamma}, x^{k}\right) x_{\alpha}^{i}+F\left(t^{\gamma}, x^{k}\right),
$$

where $h_{\alpha \beta}\left(t^{\gamma}\right)$ (resp., $g_{i j}\left(x^{k}\right)$ ) is a semi-Riemannian metric on the temporal (resp., spatial) manifold $T$ (resp., $M), U_{(\alpha)}^{(i)}\left(t^{\gamma}, x^{k}\right)$ are the local components of a distinguished tensor on $J^{1}(T, M)$ and $F\left(t^{\gamma}, x^{k}\right)$ is a smooth function on $T \times M$, is called an $a u$ tonomous metrical multi-time Lagrange space of electrodynamics (EDML).

REMARK 1.3. The nondynamical character (i.e., the independence with respect to the temporal coordinates) of the spatial metric $g_{i j}\left(x^{k}\right)$ determined us to use the terminology of autonomous in the previous definition.

The aim of this paper is to develop the differential geometry and the abstract field theory on $E D M L_{p}^{n}$, in the sense of $d$-connections, $d$-torsions, $d$-curvatures, generalized Maxwell equations and generalized Einstein equations.

2. The geometry of autonomous metrical multi-time Lagrange space of electrodynamics $E D M L_{p}^{n}$. In this section, we will apply the general geometrical development of a metrical multi-time Lagrange space [6], to the particular space of electrodynamics $E D M L_{p}^{n}$

To begin this development, consider the energy action functional associated to the multi-time Lagrangian of electrodynamics

$$
\mathscr{L}=L \sqrt{|h|}=\left[h^{\alpha \beta}\left(t^{\gamma}\right) g_{i j}\left(x^{k}\right) x_{\alpha}^{i} x_{\beta}^{j}+U_{(i)}^{(\alpha)}\left(t^{\gamma}, x^{k}\right) x_{\alpha}^{i}+F\left(t^{\gamma}, x^{k}\right)\right] \sqrt{|h|},
$$

namely,

$$
\mathscr{E}_{\mathscr{L}}: C^{\infty}(T, M) \longrightarrow \mathbb{R}, \quad \mathscr{E}_{\mathscr{L}}(f)=\int_{T} \mathscr{L} d t^{1} \wedge d t^{2} \wedge \cdots \wedge d t^{p},
$$


where the temporal manifold $T$ is considered compact and orientable, the local expression of the smooth map $f$ is $\left(t^{\alpha}\right) \rightarrow\left(x^{i}\left(t^{\alpha}\right)\right)$ and $x_{\alpha}^{i}=\partial x^{i} / \partial t^{\alpha}$. In this context, a general result from [6] implies the following result.

THEOREM 2.1. The extremals of the energy functional $\mathscr{E}_{L}$, associated to the multitime Lagrangian of electrodynamics $\mathscr{L}$, are equivalent with generalized harmonic maps [7] of the multi-time dependent spray $(H, G)$, defined by the temporal components

$$
H_{(\alpha) \beta}^{(i)}=-\frac{1}{2} H_{\alpha \beta}^{\gamma} x_{\gamma}^{i}
$$

and the local spatial components

$$
G_{(\alpha) \beta}^{(i)}=\frac{1}{2} \gamma_{j k}^{i} x_{\alpha}^{j} x_{\beta}^{k}+\frac{h_{\alpha \beta} g^{i l}}{4 p}\left[U_{(l) m}^{(\mu)} x_{\mu}^{m}+\frac{\partial U_{(l)}^{(\mu)}}{\partial t^{\mu}}+U_{(l)}^{(\mu)} H_{\mu \gamma}^{\gamma}-\frac{\partial F}{\partial x^{l}}\right],
$$

where $H_{\alpha \beta}^{\gamma}\left(\right.$ resp., $\left.\gamma_{j k}^{i}\right)$ are the Christoffel symbols of the semi-Riemannian metric $h_{\alpha \beta}$ (resp., $\left.g_{i j}\right), p=\operatorname{dim} T$, and $U_{(i) j}^{(\alpha)}=\partial U_{(i)}^{(\alpha)} / \partial x^{j}-\partial U_{(j)}^{(\alpha)} / \partial x^{i}$. In other words, these extremals verify the generalized harmonic map equations attached to the multi-time dependent spray $(H, G)$,

$$
h^{\alpha \beta}\left\{x_{\alpha \beta}^{i}+2 H_{(\alpha) \beta}^{(i)}+2 G_{(\alpha) \beta}^{(i)}\right\}=0 .
$$

Definition 2.2. The multi-time dependent spray $(H, G)$ constructed in Theorem 2.1 is called the canonical multi-time dependent spray attached to the autonomous metrical multi-time Lagrange space of electrodynamics.

Following [6], the canonical multi-time dependent spray $(H, G)$ naturally induces a nonlinear connection $\Gamma=\left(M_{(\alpha) \beta}^{(i)}, N_{(\alpha) j}^{(i)}\right)$ on $J^{1}(T, M)$, which is called the canonical nonlinear connection of the autonomous metrical multi-time Lagrange space of electrodynamics. Thus, denoting $\varphi^{i}=h^{\alpha \beta} G_{(\alpha) \beta}^{(i)}$, we can formulate the next result.

THEOREM 2.3. The canonical nonlinear connection on $J^{1}(T, M)$, attached to the autonomous metrical multi-time Lagrange space of electrodynamics is determined by the temporal components

$$
M_{(\alpha) \beta}^{(i)}=2 H_{(\alpha) \beta}^{(i)}=-H_{\alpha \beta}^{\gamma} x_{\gamma}^{i}
$$

and the local spatial components

$$
N_{(\alpha) j}^{(i)}=\frac{\partial \varphi^{i}}{\partial x_{\gamma}^{j}} h_{\alpha \gamma}=\gamma_{j k}^{i} x_{\alpha}^{k}+\frac{h_{\alpha \gamma} \mathcal{g}^{i l}}{4} U_{(l) j}^{(\gamma)} .
$$

Now, let $\left\{\delta / \delta t^{\alpha}, \delta / \delta x^{i}, \partial / \partial x_{\alpha}^{i}\right\} \subset \mathscr{L}\left(J^{1}(T, M)\right)$ and $\left\{d t^{\alpha}, d x^{i}, \delta x_{\alpha}^{i}\right\} \subset \mathscr{L} *\left(J^{1}(T, M)\right)$ be the adapted bases of the nonlinear connection $\Gamma$, where [7]

$$
\begin{aligned}
\frac{\delta}{\delta t^{\alpha}} & =\frac{\partial}{\partial t^{\alpha}}-M_{(\beta) \alpha}^{(j)} \frac{\partial}{\partial x_{\beta}^{j}}, \\
\frac{\delta}{\delta x^{i}} & =\frac{\partial}{\partial x^{i}}-N_{(\beta) i}^{(j)} \frac{\partial}{\partial x_{\beta}^{j}}, \\
\delta x_{\alpha}^{i} & =d x_{\alpha}^{i}+M_{(\alpha) \beta}^{(i)} d t^{\beta}+N_{(\alpha) j}^{(i)} d x^{j} .
\end{aligned}
$$


Following the general exposition from [6], by a direct calculation, we can determine the adapted components of the generalized Cartan canonical connection of the autonomous metrical multi-time Lagrange space of electrodynamics, together with its torsion and curvature adapted local $d$-tensors.

THEOREM 2.4. (i) The generalized Cartan canonical connection

$$
C \Gamma=\left(H_{\alpha \beta}^{\gamma}, G_{j y}^{k}, L_{j k}^{i}, C_{j(k)}^{i(\gamma)}\right)
$$

of the autonomous metrical multi-time Lagrange space of electrodynamics has the adapted coefficients

$$
H_{\alpha \beta}^{\gamma}=H_{\alpha \beta}^{\gamma}, \quad G_{j \gamma}^{k}=0, \quad L_{j k}^{i}=\gamma_{j k}^{i}, \quad C_{j(k)}^{i(\gamma)}=0 .
$$

(ii) The torsion $\mathrm{T}$ of the generalized Cartan canonical connection of the autonomous metrical multi-time Lagrange space of electrodynamics is determined by three local adapted d-tensors, namely,

$$
\begin{aligned}
& R_{(\mu) \alpha \beta}^{(m)}=-H_{\mu \alpha \beta}^{\gamma} x_{\gamma}^{m}, \\
& R_{(\mu) \alpha j}^{(m)}=-\frac{h_{\mu \eta} g^{m k}}{4}\left[H_{\alpha \gamma}^{\eta} U_{(k) j}^{(\gamma)}+\frac{\partial U_{(k) j}^{(\eta)}}{\partial t^{\alpha}}\right], \\
& R_{(\mu) i j}^{(m)}=r_{i j k}^{m} x_{\mu}^{k}+\frac{h_{\mu \eta} g^{m k}}{4}\left[U_{(k) i \mid j}^{(\eta)}+U_{(k) j \mid i}^{(\eta)}\right],
\end{aligned}
$$

where $H_{\mu \alpha \beta}^{\gamma}\left(\right.$ resp., $\left.r_{i j k}^{m}\right)$ are the local curvature tensors of the semi-Riemannian metric $h_{\alpha \beta}$ (resp., $g_{i j}$ ) and " $i$ " represents the local spatial horizontal covariant derivative induced by the generalized Cartan connection (see [6]).

(iii) The curvature $\mathbf{R}$ of the generalized Cartan canonical connection of the autonomous metrical multi-time Lagrange space of electrodynamics is determined by two local adapted d-tensors, namely, $H_{\alpha \beta \gamma}^{\eta}$ and $R_{i j k}^{l}=r_{i j k}^{l}$, that is, exactly the curvature tensors of the semi-Riemannian metrics $h_{\alpha \beta}$ and $g_{i j}$.

3. Generalized Maxwell equations on $E D M L_{p}^{n}$. To describe the generalized electromagnetism theory on the autonomous metrical multi-time Lagrange space, consider the canonical Liouville $d$-vector field $\mathbf{C}=x_{\alpha}^{i} \partial / \partial x_{\alpha}^{i}$ on $J^{1}(T, M)$, and construct the metrical deflection d-tensors [5]

$$
\begin{aligned}
\bar{D}_{(i) \beta}^{(\alpha)} & =\left[h^{\alpha \mu} g_{i m} x_{\mu}^{m}\right]_{/ \beta}=0, \\
D_{(i) j}^{(\alpha)} & =\left[h^{\alpha \mu} g_{i m} x_{\mu}^{m}\right]_{\mid j}=-\frac{1}{4} U_{(i) j}^{(\alpha)}, \\
d_{(i)(j)}^{(\alpha)(\beta)} & =\left.\left[h^{\alpha \mu} g_{i m} x_{\mu}^{m}\right]\right|_{(j)} ^{(\beta)}=h^{\alpha \beta} g_{i j},
\end{aligned}
$$

where " $/ \beta$ ", “ $\mid j$ ", and “ $\left.\right|_{(j)} ^{(\beta)}$ " are the local covariant derivatives induced by the generalized Cartan canonical connection $C \Gamma$ (see also [6]).

Taking into account the general expressions of the local electromagnetic d-tensors of a metrical multi-time Lagrange space [5], by a direct calculation, we deduce the following result. 
Proposition 3.1. The local electromagnetic d-tensors of the autonomous metrical multi-time Lagrange space of electrodynamics have the expressions,

$$
\begin{gathered}
F_{(i) j}^{(\alpha)}=\frac{1}{2}\left[D_{(i) j}^{(\alpha)}-D_{(j) i}^{(\alpha)}\right]=\frac{1}{8}\left[U_{(j) i}^{(\alpha)}-U_{(i) j}^{(\alpha)}\right]=-\frac{1}{4} U_{(i) j}^{(\alpha)} \\
f_{(i)(j)}^{(\alpha)(\beta)}=\frac{1}{2}\left[d_{(i)(j)}^{(\alpha)(\beta)}-d_{(j)(i)}^{(\alpha)(\beta)}\right]=0 .
\end{gathered}
$$

Particularizing the generalized Maxwell equations of multi-time electromagnetic field, described in the general context of a metrical multi-time Lagrange space [5], we deduce the main result of the generalized electromagnetism on the autonomous metrical multi-time Lagrange space of electrodynamics.

THEOREM 3.2. The electromagnetic local components $F_{(i) j}^{(\alpha)}$ of the autonomous metrical multi-time Lagrange space of electrodynamics are governed by the following generalized Maxwell equations,

$$
F_{(i) j / \beta}^{(\alpha)}=\frac{1}{2} \mathscr{A}_{\{i, j\}} h^{\alpha \mu} g_{i m} R_{(\mu) \beta j}^{(m)}, \quad \sum_{\{i, j, k\}} F_{(i) j \mid k}^{(\alpha)}=0,\left.\quad \sum_{\{i, j, k\}} F_{(i) j}^{(\alpha)}\right|_{(k)} ^{(\gamma)}=0,
$$

where $\mathscr{A}_{\{i, j\}}$ represents an alternate sum and $\sum_{\{i, j, k\}}$ means a cyclic sum.

4. Generalized Einstein equations and conservation laws on $E D M L_{p}^{n}$. To start the development of the generalized gravitational theory on the autonomous metrical multi-time Lagrange space of electrodynamics $E D M L_{p}^{n}$, we point out that the vertical metrical $d$-tensor $G_{(i)(j)}^{(\alpha)(j)}=h^{\alpha \beta}(t) g_{i j}(x)$ and the canonical nonlinear connection $\Gamma=$ $\left(M_{(\alpha) \beta}^{(i)}, N_{(\alpha) j}^{(i)}\right)$ of this space induce a natural multi-time gravitational potential (i.e., $a$ Sasakian like-metric) on the 1-jet space $J^{1}(T, M)$, which is expressed by [5]

$$
G=h_{\alpha \beta} d t^{\alpha} \otimes d t^{\beta}+g_{i j} d x^{i} \otimes d x^{j}+h^{\alpha \beta} g_{i j} \delta x_{\alpha}^{i} \otimes \delta x_{\beta}^{j} .
$$

Consider $C \Gamma=\left(H_{\alpha \beta}^{\gamma}, 0, \gamma_{j k}^{i}, 0\right)$ the generalized Cartan canonical connection of $E D M L_{p}^{n}$.

We postulate that the generalized Einstein equations which govern the gravitational $h$-potential $G$ of the metrical multi-time Lagrange space of electrodynamics $E D M L_{p}^{n}$ are the abstract geometrical Einstein equations attached to the generalized Cartan canonical connection and the adapted metric $G$ on $J^{1}(T, M)$, that is,

$$
\operatorname{Ric}(C \Gamma)-\frac{\operatorname{Sc}(C \Gamma)}{2} G=\mathscr{K} \mathscr{T},
$$

where Ric $(C \Gamma)$ represents the Ricci $d$-tensor of the generalized Cartan connection, $\operatorname{Sc}(C \Gamma)$ is its scalar curvature, $\mathscr{K}$ is the Einstein constant and $\mathscr{T}$ is an intrinsic $d$-tensor of matter which is called the stress-energy $d$-tensor.

In the adapted basis $\left\{X_{A}\right\}=\left\{\delta / \delta t^{\alpha}, \delta / \delta x^{i}, \partial / \partial x_{\alpha}^{i}\right\}$, the curvature $d$-tensor $\mathbf{R}$ of the generalized Cartan connection is expressed locally by $\mathbf{R}\left(X_{C}, X_{B}\right) X_{A}=R_{A B C}^{D} X_{D}$. Therefore, it follows that we have $\operatorname{Ric}(C \Gamma)\left(X_{A}, X_{B}\right)=R_{A B}=R_{A B D}^{D}$ and $\operatorname{Sc}(C \Gamma)=G^{A B} R_{A B}$, where

$$
G^{A B}= \begin{cases}h_{\alpha \beta}, & \text { for } A=\alpha, B=\beta, \\ g^{i j}, & \text { for } A=i, B=j, \\ h_{\alpha \beta} g^{i j}, & \text { for } A={ }_{(\alpha)}^{(i)}, B={ }_{(\beta)}^{(j)}, \\ 0, & \text { otherwise. }\end{cases}
$$


Taking into account the expressions of the local curvature $d$-tensors of the generalized Cartan connection of $E D M L_{p}^{n}$, by computations, we deduce the following theorem.

THEOREM 4.1. The Ricci d-tensor Ric $(C \Gamma)$ of the autonomous metrical multi-time Lagrange space of electrodynamics is characterized by two effective adapted local Ricci d-tensors, namely, $H_{\alpha \beta}$ and $R_{i j}=r_{i j}$, where $H_{\alpha \beta}$ (resp., $r_{i j}$ ) are the local Ricci tensors associated to the semi-Riemannian metric $h_{\alpha \beta}\left(\right.$ resp., $\left.g_{i j}\right)$.

Consequently, denoting $H=h^{\alpha \beta} H_{\alpha \beta}, R=g^{i j} R_{i j}$, by direct calculations, we obtain the following theorem.

THEOREM 4.2. The scalar curvature Sc $(C \Gamma)$ of the generalized Cartan connection of $E D M L_{p}^{n}$ is given by

$$
\operatorname{Sc}(C \Gamma)=H+R=H+r,
$$

where $H$ and $r$ are the scalar curvatures of the semi-Riemannian metrics $h$ and $g$.

In conclusion, we can establish the main result of the gravitational theory on $E D M L_{p}^{n}$.

THEOREM 4.3. The local generalized Einstein equations which govern the multi-time gravitational potential $G$, induced by the Lagrangian function of autonomous metrical multi-time Lagrange space of electrodynamics, have the form

$$
\begin{gathered}
H_{\alpha \beta}-\frac{H+r}{2} h_{\alpha \beta}=\mathscr{K} \mathcal{T}_{\alpha \beta}, \\
r_{i j}-\frac{H+r}{2} g_{i j}=\mathscr{K} \mathcal{T}_{i j}, \\
-\frac{H+r}{2} h^{\alpha \beta} g_{i j}=\mathscr{K}_{(i)(j)}^{(\alpha)(\beta)}, \\
0=\mathscr{T}_{\alpha i}, \quad 0=\mathscr{T}_{i \alpha}, \quad 0=\mathscr{T}_{(i) \beta}^{(\alpha)}, \\
0=\mathscr{T}_{\alpha(i)}^{(\beta)}, \quad 0=\mathscr{F}_{i(j)}^{(\alpha)}, \quad 0=\mathscr{T}_{(i) j}^{(\alpha)},
\end{gathered}
$$

where $\mathscr{T}_{A B}, A, B \in\left\{\alpha, i,{ }_{(i)}^{(\alpha)}\right\}$, are the adapted components of the stress-energy distinguished tensor $\mathcal{T}$.

REMARK 4.4. Assuming $p=\operatorname{dim} T>2$ and $n=\operatorname{dim} M>2$, the set (4.5) of the generalized Einstein equations can be rewritten in the classical form

$$
H_{\alpha \beta}-\frac{H}{2} h_{\alpha \beta}=\mathscr{K} \tilde{\mathcal{T}}_{\alpha \beta}, \quad r_{i j}-\frac{r}{2} g_{i j}=\mathscr{K} \tilde{\mathcal{T}}_{i j},
$$

where $\tilde{\mathscr{T}}_{A B}, A, B \in\{\alpha, i\}$ are the adapted local components of a new stress-energy $d$ tensor $\tilde{\mathcal{T}}$. This new form of the Einstein equations is deeply treated in the more general case of a generalized metrical multi-time Lagrange space [3].

Note that, in order to have the compatibility of the Einstein equations, it is necessary that the certain adapted local components of the stress-energy $d$-tensor vanish a priori. At the same time, it is well known that, from a physical point of view, the 
stress-energy $d$-tensor $\mathscr{T}$ must verify the local generalized conservation laws $\mathscr{T}_{A \mid B}^{B}=0$, for all $A \in\left\{\alpha, i,{ }_{(i)}^{(\alpha)}\right\}$, where $\mathscr{T}_{A}^{B}=G^{B D} \mathscr{T}_{D A}$. In this context, by direct computations, we obtain the following result.

THEOREM 4.5. The generalized conservation laws of the generalized Einstein equations of the multi-time gravitational potential of autonomous metrical multi-time Lagrange space are given by

$$
\left[H_{\beta}^{\mu}-\frac{H+r}{2} \delta_{\beta}^{\mu}\right]_{/ \mu}=0, \quad\left[r_{j}^{m}-\frac{H+r}{2} \delta_{j}^{m}\right]_{\mid m}=0,
$$

where $H_{\beta}^{\mu}=h^{\mu v} H_{v \beta}$ and $r_{j}^{m}=g^{m s} r_{s j}$.

REMARK 4.6. Taking into account the components $\tilde{\mathscr{T}}_{\alpha \beta}$ and $\tilde{\mathscr{T}}_{i j}$ of the new stressenergy $d$-tensor $\tilde{\mathscr{T}}$ appeared in the classical form (4.8) of the generalized Einstein equations, the generalized conservation laws modify in the classical form.

ACKNowledgement. Many thanks go to Prof Dr. V. Balan, whose suggestions upon a previous version of this paper were very useful.

\section{REFERENCES}

[1] N. Kamran and P. J. Olver, Le problème d'équivalence à une divergence près dans le calcul des variations des intégrales multiples. [The problem of equivalence up to a divergence in the calculus of variations for multiple integrals], C. R. Acad. Sci. Paris Sér. I Math. 308 (1989), no. 8, 249-252 (French).

[2] R. Miron and M. Anastasiei, The Geometry of Lagrange Spaces: Theory and Applications, Fundamental Theories of Physics, vol. 59, Kluwer, Dordrecht, 1994.

[3] M. Neagu, Generalized metrical multi-time Lagrange geometry of physical field, Forum Mathematicum, Journals from de Gruyter, in press, http://xxx.lanl.gov/abs/math. DG/0011003, 2001.

[4] _ , The geometry of relativistic rheonomic Lagrange spaces, Workshop on Differential Geometry, Global Analysis, Lie Algebras, Aristotle University of Thessaloniki, Greece, 1997 (G. Tsagas, ed.), Balkan Society of Geometers Proceedings, vol. 5, Geometry Balkan Press, Bucharest, 2001, pp. 142-168.

[5]__ Riemann-Lagrange geometrical background for multi-time physical fields, Balkan J. Geom. Appl. 6 (2001), no. 2, 49-70.

[6] M. Neagu and C. Udriste, The geometry of metrical multi-time Lagrange spaces, http:// xxx.lanl.gov/abs/math.DG/0009071, 2000.

[7]__ Multi-time dependent sprays and harmonic maps on $J^{1}(T, M)$, Third Conference of Balkan Society of Geometers, Politehnica University of Bucharest, Romania, 2000, http://xxx.lanl.gov/abs/math.DG/0009049.

[8] P. J. Olver, Applications of Lie Groups to Differential Equations, Graduate Texts in Mathematics, vol. 107, Springer-Verlag, New York, 1986.

Mircea Neagu: Department of Mathematics I, University Politehnica of Bucharest, SPLAIUL INDEPENDENTEI 313-77206, BUCHAREST, ROMANIA

E-mail address: mi rcea@mathem. pub.ro 


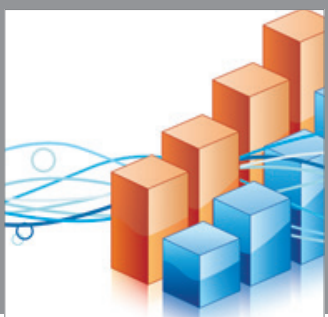

Advances in

Operations Research

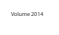

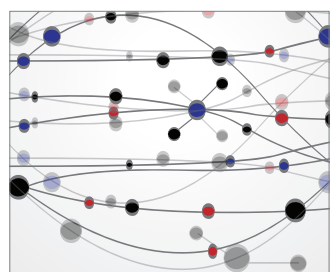

\section{The Scientific} World Journal
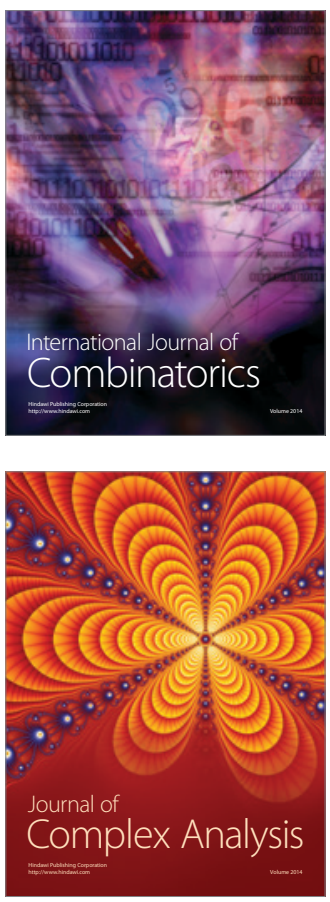

International Journal of

Mathematics and

Mathematical

Sciences
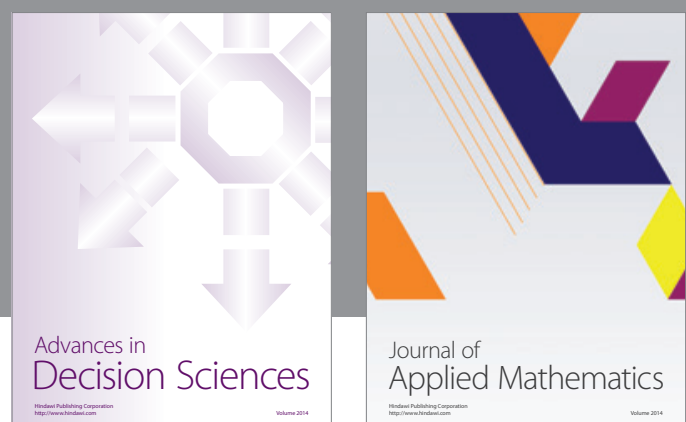

Journal of

Applied Mathematics
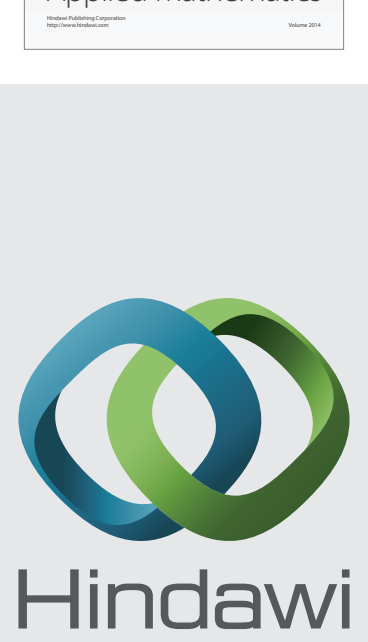

Submit your manuscripts at http://www.hindawi.com
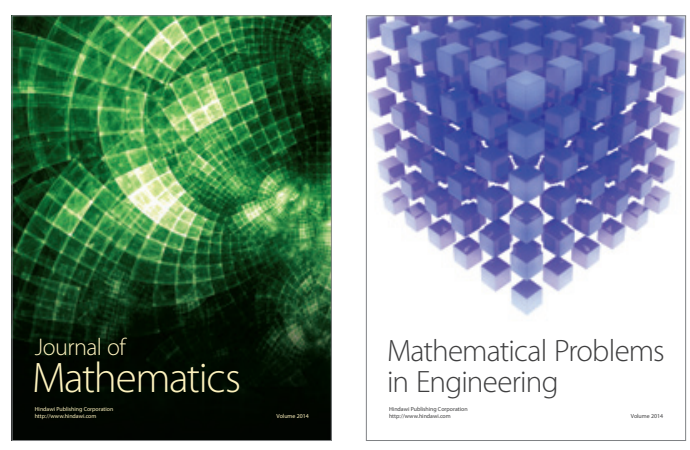

Mathematical Problems in Engineering
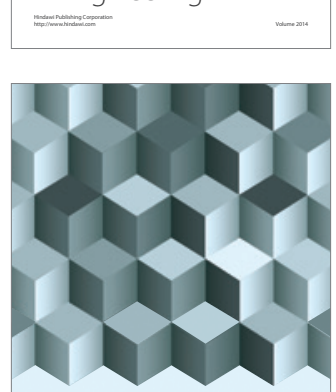

Journal of

Function Spaces
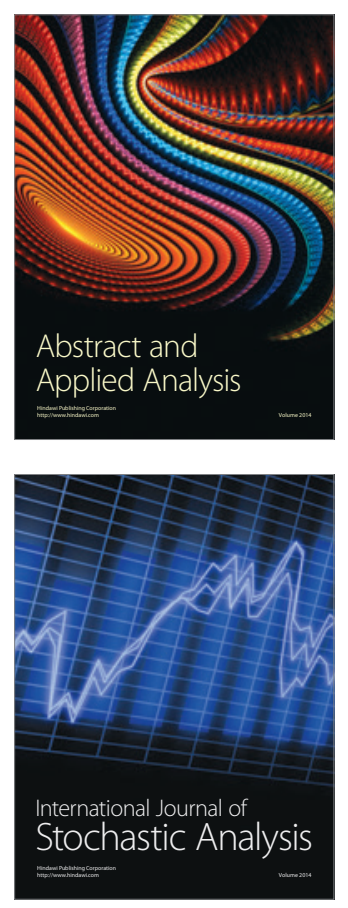

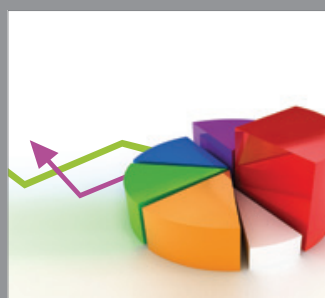

ournal of

Probability and Statistics

Promensencen
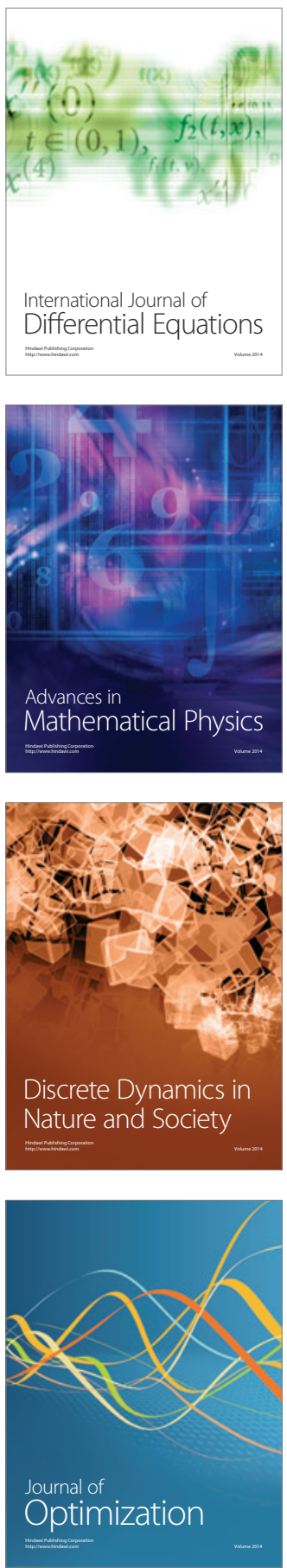